\title{
Hydatid Cyst
}

National Cancer Institute

\section{Source}

National Cancer Institute. Hydatid Cyst. NCI Thesaurus. Code C97063.

A cyst that is attached to the fallopian tube and originates from the remnants of the Mullerian duct. 